\title{
Administrata lokale dhe demografia e kazasë së Elbasanit, sipas vjetarëve të Manastirit, për periudhën 1888-1896
}

Ermal Nurja

Fryma e re reformatore mbi të cilën u ngrit riorganizimi i shtetit osman, pas shpalljes së Tanzimatit, mes të tjerash do të ndikonte pa dyshim edhe në botën e botimeve (gazeta, revista, libra, kalendarë).

Vjetari i parë u botua në vitin 1263 (1847) dhe ishte vjetari statistikor i Perandorisë Osmane. Ky botim u ndoq me kureshtje në perëndim, prandaj edhe statistika e parë e Perandorisë Osmane, u përkthye në frëngjisht dhe $\mathrm{u}$ botua menjëherë nga Bianchi në formë fashikujsh në revistën Journal asiatique, për t'u botuar më pas në vitin 1848, në formën e një libri nën titullin " $L e$ premier annuaire imperial de l'Empire ottoman au tableau de l'etat politique, civil, militaire, juridiciaire et administratif de la Turquie" (Paris 1848).

Përveç vjetarëve shtetërore dhe atyre private edhe pushteti lokal, në rang vilajeti botuan vjetarët e tyre. Deri më sot, kanë mbërritur 504 vjetarë të vilajete të ndryshme të ish-Perandorisë Osmane. Për trevat tona shqiptare, ne deri më sot njohim 7 vjetarë të vilajetit të Manastirit, 8 vjetarë të vilajetit të Kosovës, 5 vjetarë të vilajetit të Shkodrës, 8 vjetarë të vilajetit të Janinës dhe 2 vjetarë të vilajetit të Prizrenit.

Në këtë artikull, ne jemi bazuar vetëm te shtatë vjetarët osmanë që u hartuan pas ndarjes së fundit administrative në vilajetin e madh të Rumelisë, si rezultat i së cilës, Elbasani u shndërrua në qendër sanxhaku nën varësinë administrative të vilajetit të Manastirit. Në hartimin e tabelave janë përdorur numrat romakë për të dalluar vjetarët njërin nga tjetri. Shkurtimisht, të dhënat për sanxhakun e Elbasanit, në secilin prej vjetarëve, gjendet më poshtë: 


\section{Albanon}

\section{Revistë kulturore}

Vjetari I: Ky vjetar i përket vitit 1305 (1888). Kapitulli për sanxhakun e Elbasanit gjendet në faqet 220-222.

Vjetari II: Ky vjetar i përket vitit 1308 (1891). Kapitulli për sanxhakun e Elbasanit gjendet në faqet 200-207.

Vjetari III: Ky vjetar i përket vitit 1310 (1892). Kapitulli për sanxhakun e Elbasanit gjendet në faqet 288-297 të këtij vjetari.

Vjetari IV: Ky vjetar i përket vitit 1311 (1893). Kapitulli për sanxhakun e Elbasanit gjendet në faqet 270-275.

Vjetari V: Ky vjetar i përket vitit 1312 (1894). Kapitulli për sanxhakun e Elbasanit gjendet në faqet 284-288.

Vjetari VI: Ky vjetar i përket vitit 1313 (1895). Kapitulli për sanxhakun e Elbasanit gjendet në faqet 160-164.

Vjetari VII: Ky vjetar i përket vitit 1314 (1896). Kapitulli për sanxhakun e Elbasanit gjendet në faqet 162-166 të këtij vjetari.

Këshilli administrativ i sanxhakut të Elbasanit formohej nga anëtarët, që kishin post për shkak të detyrës që mbulonin siç ishin: mytesarifi, kadiu, llogaritari i sanxhakut, drejtori i protokollit të sanxhakut (këta ishin edhe figurat qendore të pushtetit lokal të sanxhakut të Elbasanit) dhe anëtarëve që përzgjidheshin nga popullsia vendase. Sekretari i këshillit administrativ me përjashtim të vitit 1888 ishte Faiku ${ }^{1}$, ndërsa për gjithë periudhën tjetër, sekretar ishte Mustafa Lytfi.

Në vjetarin I, antarët e zgjedhur janë: Shehsyvar ${ }^{2}$, Hysen, Jovan Xhufka, Nikolla Papajani; në vjetarin II: Hasan, Nikolla Papajani, Vasil Popa; në vjetarin III: Aqif', Hysen Hysni, Nikolla Papajani, Vasil Popa; në vjetarin IV: Aqif, Hysen Hysni, Nos Mim Nosi, Vasil Popa; në vjetarin V: Adil, Hysen Hysni, Nikolla Papajani, Nosi Mim Nos; në vjetarin VI: Adil bej, Hysen Hysni, Nikolla Papajani, Vasil Popa; në vjetarin e VII: Hasan aga, Vasil Popa.

1 Duke qenë se për kohën kanë qenë figura të spikatura e tepër të njohura, shpesh nëpër vjetarë ata jepen vetëm si emra, pa mbiemrat e tyre, edhe pse sot nuk mund të identifikohen të plotë emrat e tyre. (shën. E. N.)

2 Nga familja Verlaci

3 Kupto: Aqif Pashë Elbasani 
Këshilli administrativ i sanxhakut të Elbasanit

\begin{tabular}{|l|c|c|c|c|c|c|c|}
\hline Titulli & I & II & III & IV & V & VI & VII \\
\hline Mytesarif & Vehbi & Junuz Zyhdi & Junuz Zyhdi & Junuz Zyhdi & Junuz Zyhdi & Junuz Zyhdi & Junuz Zyhdi \\
\hline Kadiu & Numan & Ahmet Fazlli & Abdylmuin & Abdylmuin & Abdylmuin & $\begin{array}{c}\text { Adem } \\
\text { Safijudin }\end{array}$ & $\begin{array}{c}\text { Adem } \\
\text { Safijudin }\end{array}$ \\
\hline $\begin{array}{l}\text { Llogaritari i } \\
\text { sanxhakut }\end{array}$ & Ethem & Ethem Sabri & Salih & Salih & Salih & Salih & Salih \\
\hline $\begin{array}{l}\text { Drejtori i } \\
\text { protokollit }\end{array}$ & Xhelal & Nexhmedin & Nexhmedin & Nexhmedin & Nexhmedin & Nexhmedin & Nexhmedin \\
\hline
\end{tabular}

Zyra e llogaritarit të sanxhakut të Elbasanit

\begin{tabular}{|c|c|c|c|c|c|c|c|}
\hline & I & II & III & IV & V & VI & VII \\
\hline $\begin{array}{l}\text { Ndihmës } \\
\text { llogaritari }\end{array}$ & Murat & Murat & Murat & Murat & Murat & Murat & Murat \\
\hline $\begin{array}{l}\text { Sekretari i } \\
\text { sanxhakut }\end{array}$ & $X X X^{1}$ & $X X X$ & Abdurrahim & Abdurrahim & Abdurrahim & Abdurrahim & Abdurrahim \\
\hline $\begin{array}{l}\text { Shoqërues i } \\
\text { sekretarit të } \\
\text { sanxhakut }\end{array}$ & $X X X$ & $X X X$ & Mehmet Ali & Mehmet Ali & Mehmet Ali & Mehmet Ali & Mehmet Ali \\
\hline $\begin{array}{l}\text { Sekretari i } \\
\text { qendrës së } \\
\text { kazasë }\end{array}$ & Ymer & XXX & Ymer & Ymer & Ymer & Ymer & Ymer \\
\hline $\begin{array}{l}\text { Shoqëruesi i } \\
\text { sekretarit të } \\
\text { kazasë }\end{array}$ & $X X X$ & $X X X$ & Mustafa & Mustafa & Mustafa & Rexhep & Rexhep \\
\hline $\begin{array}{l}\text { Sekretari i } \\
\text { çështjeve ditore }\end{array}$ & Jusuf & Ali & Ryzhdi & Ryzhdi & Izet & Veli & Veli \\
\hline $\begin{array}{l}\text { Regjistruesi nga } \\
\text { keqshkronja në } \\
\text { të pastër }\end{array}$ & XXX & Mihal & Ismail & Ismail & Abdurrahim & Abdurrahim & Halili \\
\hline $\begin{array}{l}\text { Regjistruesi nga } \\
\text { keqshkronja në } \\
\text { të pastër }\end{array}$ & $X X X$ & $X X X$ & Abdurrahim & Abdylhalimi & Veli & Demiri & Demiri \\
\hline Arkëtari & $X X X$ & Sylejman & Fejzullah & Fejzullah & Sylejman & Sylejman & Fejzullah \\
\hline $\begin{array}{l}\text { Regjistruesi i të } \\
\text { ardhurave }\end{array}$ & Myhtar & Ymer & XXX & $X X X$ & $X X X$ & $X X X$ & $X X X$ \\
\hline $\begin{array}{l}\text { Regjistruesi i } \\
\text { shpenzimeve }\end{array}$ & Sylejman & Abdurrahim & $X X X$ & $X X X$ & $X X X$ & $X X X$ & $X X X$ \\
\hline
\end{tabular}

1 Shenja XXX kupton që në atë vit nuk ka pasur të zgjedhur në atë funksion, ose nuk ka funksionuar si post. 


\section{Albanon}

\section{Revistë kulturore}

Zyra e protokollit të sanxhakut

\begin{tabular}{|l|c|c|c|c|c|c|c|}
\hline & I & II & III & IV & V & VI & VII \\
\hline $\begin{array}{l}\text { Zëvendës drejtori i zyrës së } \\
\text { protokollit të sanxhakut }\end{array}$ & Ibrahim & Ibrahim & Ibrahim & Ibrahim & Ibrahim & Ibrahim & Ibrahim \\
\hline $\begin{array}{l}\text { Nëpunësi i } \\
\text { dokumentacioneve }\end{array}$ & Bahaudin & Hysen & Hysen Zihni & Hysen Zihni & Hysen Vehbi & Hysen Zihni & Hysen Zihni \\
\hline $\begin{array}{l}\text { Sekretari i parë për } \\
\text { zbardhjen e keqashkronjës }\end{array}$ & Hysen & Hysen & Hysen & Hysen & Hysen & Hysen & Hysen \\
\hline $\begin{array}{l}\text { Sekretari idytë për zbardhjen e } \\
\text { keqashkronjës }\end{array}$ & Hysen & Said & Said & Said & Said & Said & Said \\
\hline
\end{tabular}

Zyra e gjendjes civile

\begin{tabular}{|l|c|c|c|c|c|c|c|}
\hline & I & II & III & IV & V & VI & VII \\
\hline Nëpunësi i gjendjes civile & XXX & Selman & Selman & Selman & Selman & Selman & Selman \\
\hline Sekretari i gjendjes civile & XXX & Ymer & Ymer & Ymer & Ymer & Ymer & Ymer \\
\hline
\end{tabular}

Zyra e taksave (vergjive)

\begin{tabular}{|l|c|c|c|c|c|c|c|}
\hline & I & II & III & IV & V & VI & VII \\
\hline Nëpunësi i zyrës së taksave & XXX & XXX & Xhemil & Xhemil & Abdullah & Abdullah & Abdullah \\
\hline Kryesekretari & XXX & XXX & Mustafa & Mustafa Naili & Mustafa & Mustafa & Mustafa \\
\hline Shoqëruesi i sekretarit & XXX & XXX & Ibrahim & Ibrahim & Ibrahim & Ibrahim & Ibrahim \\
\hline Defterxhiu & XXX & XXX & Besim & Besim & Besim & Besim & Besim \\
\hline
\end{tabular}

Zyra e pronave (tapive)

\begin{tabular}{|l|c|c|c|c|c|c|c|}
\hline & I & II & III & IV & V & VI & VII \\
\hline Nëpunësi i zyrës së tapive & Salih & Halil & Halil & Halil & Mihal & Sadik & Sadik \\
\hline Kryesekretari & Mustafa & Mustafa & Mustafa & Mustafa & Mustafa & Mustafa & Mehmed \\
\hline Sekretari i tapive & Halim & Halim & Halim & Halim & Halim & Halim & Halim \\
\hline Taksidari & XXX & XXX & Ibrahim & Ibrahim & Ibrahim & Ibrahim & Ibrahim \\
\hline
\end{tabular}

Gjykata e shkallës së parë të Elbasanit

\begin{tabular}{|l|c|c|c|c|c|c|c|}
\hline & I & II & III & IV & V & VI & VII \\
\hline Kryetari i gjykatës & $\begin{array}{c}\text { Numan } \\
\text { (kadiu) }\end{array}$ & $\begin{array}{c}\text { Ahmet Fazlli } \\
\text { (kadiu) }\end{array}$ & Abdylmuin & Abdylmuin & Abdylmuin & Adem Vasfi & $\begin{array}{c}\text { Adem } \\
\text { Safijudin }\end{array}$ \\
\hline Kryetari i penales & $\begin{array}{c}\text { Osman } \\
\text { Tevfik }\end{array}$ & $\begin{array}{c}\text { Osman } \\
\text { Tevfik }\end{array}$ & Osman Tevfik & $\begin{array}{c}\text { Osman } \\
\text { Tevfik }\end{array}$ & $\begin{array}{c}\text { Osman } \\
\text { Tevfik }\end{array}$ & $\begin{array}{c}\text { Osman } \\
\text { Tevfik }\end{array}$ & $\begin{array}{c}\text { Osman } \\
\text { Tevfik }\end{array}$ \\
\hline Anëtari gjykatës & Haxhi Ali & Haxhi Ali & Haxhi Ali & Haxhi Ali & Haxhi Ali & Haxhi Ali & Mehmed \\
\hline
\end{tabular}




\begin{tabular}{|l|c|c|c|c|c|c|c|}
\hline & I & II & III & IV & V & VI & VII \\
\hline Anëtar i gjykatës & Kostantin & Kostantin & Kostantin & Kostantin & Kostantin & $\begin{array}{c}\text { Spiro } \\
\text { Xhufka }\end{array}$ & $\begin{array}{c}\text { Spiro } \\
\text { Xhufka }\end{array}$ \\
\hline Mylazimi i gjykatës & Ymer & Alush & Ali Ryzhdi & Ali Ryzhdi & Ali Ryzhdi & Ali Ryzhdi & Mahmut \\
\hline Kryesekretari & $\begin{array}{c}\text { Mehmet } \\
\text { Zyhdi }\end{array}$ & $\begin{array}{c}\text { Mehmet } \\
\text { Zyhdi }\end{array}$ & $\begin{array}{c}\text { Mehmet } \\
\text { Zyhdi }\end{array}$ & $\begin{array}{c}\text { Mehmet } \\
\text { Zyhdi }\end{array}$ & $\begin{array}{c}\text { Mehmet } \\
\text { Zyhdi }\end{array}$ & $\begin{array}{c}\text { Mehmet } \\
\text { Zyhdi }\end{array}$ & $\begin{array}{c}\text { Mehmet } \\
\text { Zihni }\end{array}$ \\
\hline $\begin{array}{l}\text { Sekretari i akteve të } \\
\text { gjykatës }\end{array}$ & $\begin{array}{c}\text { Mehmet } \\
\text { Nuri }\end{array}$ & $\begin{array}{c}\text { Mehmet } \\
\text { Nuri }\end{array}$ & $\begin{array}{c}\text { Mahmut } \\
\text { Besim }\end{array}$ & $\begin{array}{c}\text { Mahmut } \\
\text { Besim }\end{array}$ & $\begin{array}{c}\text { Mahmut } \\
\text { Besim }\end{array}$ & $\begin{array}{c}\text { Mahmut } \\
\text { Besim }\end{array}$ & Jusuf \\
\hline $\begin{array}{l}\text { Sekretari iakteve të } \\
\text { gjykatës }\end{array}$ & Alush & Jusuf & Jusuf & Jusuf & Jusuf & Jusuf & Musa \\
\hline $\begin{array}{l}\text { Sekretari i akteve të } \\
\text { gjykatës }\end{array}$ & $\begin{array}{c}\text { Mehmet } \\
\text { Besim }\end{array}$ & $\begin{array}{c}\text { Mehmet } \\
\text { Besim }\end{array}$ & Jusuf & Jusuf & Jusuf & Jusuf & XXX \\
\hline $\begin{array}{l}\text { Sekretari i akteve të } \\
\text { gjykatës }\end{array}$ & Mihal & XXX & XXX & XXX & XXX & XXX \\
\hline $\begin{array}{l}\text { Sekretari i gjykatës së } \\
\text { sherijes }\end{array}$ & Mustafa & XXX & Ibrahim & Ibrahim & Ibrahim & Ibrahim & Ibrahim \\
\hline $\begin{array}{l}\text { Prokurori i gjykatës } \\
\text { Hasan Fehmi }\end{array}$ & $\begin{array}{c}\text { Mehmet } \\
\text { Sadik }\end{array}$ & Qamil & Qamil & Malik & Qazim & Qazim \\
\hline $\begin{array}{l}\text { Nëpunësi i përmbarimit } \\
\text { gjykatës }\end{array}$ & Ismail Haki & Kapllan & Kapllan & Mihali & Mihali & Ali & Ali Ryzhdi \\
\hline
\end{tabular}

Drejtoria e policisë

\begin{tabular}{|l|c|c|c|c|c|c|c|}
\hline & I & II & III & IV & V & VI & VII \\
\hline Komisar & XXX & XXX & Hasan & Halit Nijazi & Mihal & Mihal & Mihal \\
\hline Polic & XXX & $X X X$ & Ali Riza & Ali Riza & Ali Riza & Ali Riza & Ali Riza \\
\hline Polic & $X X X$ & $X X X$ & Ali Nuri & Ali Nuri & Ali Nuri & Nuri & Ali Nuri \\
\hline Polic & $X X X$ & $X X X$ & Junus & Mehmet Zija & Junus & Junus & Junus \\
\hline Polic & $X X X$ & $X X X$ & Ahmet hafiz & XXX & Ahmet Faik & XXX & XXX \\
\hline Polic & $X X X$ & $X X X$ & Salih & $X X X$ & $X X X$ & $X X X$ & XXX \\
\hline
\end{tabular}

Drejtoria e postë-telegrafit

\begin{tabular}{|l|c|c|c|c|c|c|c|}
\hline & I & II & III & IV & V & VI & VII \\
\hline Drejtori i postë-telegrafit & $\mathrm{XXX}$ & $\mathrm{XXX}$ & Ali & Ali & Mihal & Tahsin & Tahsin \\
\hline $\begin{array}{l}\text { Nëpunësi i ndërlidhjes } \\
\text { telegrafike }\end{array}$ & $\mathrm{XXX}$ & $\mathrm{XXX}$ & $\begin{array}{c}\text { Hysen } \\
\text { Hysni }\end{array}$ & Ismail & Ismail & Ismail & Ismail \\
\hline $\begin{array}{l}\text { Nëpunësi i ndërlidhjes } \\
\text { telegrafike }\end{array}$ & $\mathrm{XXX}$ & $\mathrm{XXX}$ & Sadik & Sadik & Sadik & Rifat & Shykri \\
\hline
\end{tabular}




\section{Albanon}

\section{Revistë kulturore}

Komisioni i punëve publike

\begin{tabular}{|c|c|c|c|c|c|c|c|}
\hline & I & II & III & IV & V & VI & VII \\
\hline Kryetari & Mytesarifi & Mytesarifi & Mytesarifi & Mytesarifi & Mytesarifi & Mytesarifi & Mytesarifi \\
\hline Anëtar & $X X X$ & $X X X$ & $\begin{array}{c}\text { Shehsyvar, } \\
\text { kryetarii } \\
\text { bashkisë }\end{array}$ & $\begin{array}{c}\text { Shehsyvar, } \\
\text { kryetari i } \\
\text { bashkisë }\end{array}$ & $\begin{array}{c}\text { Shehsyvar, } \\
\text { kryetarii } \\
\text { bashkisë }\end{array}$ & $\begin{array}{c}\text { Shehsyvar, } \\
\text { kryetari i } \\
\text { bashkisë }\end{array}$ & $\begin{array}{c}\text { Shehsyvar, } \\
\text { kryetari i } \\
\text { bashkisë }\end{array}$ \\
\hline Anëtar & $X X X$ & $X X X$ & $\begin{array}{c}\text { Selman, } \\
\text { nëpunësi i } \\
\text { gjendjes civile }\end{array}$ & $\begin{array}{c}\text { Selman, } \\
\text { nëpunësi i } \\
\text { gjendjes civile }\end{array}$ & $\begin{array}{c}\text { Selman, } \\
\text { nëpunësi i } \\
\text { gjendjes civile }\end{array}$ & $\begin{array}{c}\text { Selman, } \\
\text { nëpunësi i } \\
\text { gjendjes civile }\end{array}$ & $\begin{array}{c}\text { Selman, } \\
\text { nëpunësi i } \\
\text { gjendjes civile }\end{array}$ \\
\hline Anëtar & $X X X$ & $X X X$ & $\begin{array}{c}\text { Mehmet Fikri } \\
\text { nëpunësi i } \\
\text { bankës agrare }\end{array}$ & $\begin{array}{c}\text { Mehmet Fikri } \\
\text { nëpunësii } \\
\text { bankës agrare }\end{array}$ & $\begin{array}{c}\text { Mehmet Syrri } \\
\text { nëpunësi i } \\
\text { bankës agrare }\end{array}$ & $\begin{array}{c}\text { Ibrahimi, } \\
\text { nëpunësi i } \\
\text { bankës agrare }\end{array}$ & $\begin{array}{c}\text { Mehmet Syrri } \\
\text { nëpunësi i } \\
\text { bankës agrare }\end{array}$ \\
\hline Anëtar & $X X X$ & $X X X$ & $\begin{array}{l}\text { Jovakim } \\
\text { konduktori i } \\
\text { rrugëve }\end{array}$ & $\begin{array}{l}\text { Jovakim } \\
\text { konduktori i } \\
\text { rrugëve }\end{array}$ & $\begin{array}{l}\text { Jovakim } \\
\text { konduktorii } \\
\text { rrugëve }\end{array}$ & $\begin{array}{l}\text { Ahmet Refik } \\
\text { konduktori i } \\
\text { rrugëve }\end{array}$ & $\begin{array}{c}\text { Ahmet Refik } \\
\text { konduktori i } \\
\text { rrugëve }\end{array}$ \\
\hline
\end{tabular}

Këshilli bashkiak

\begin{tabular}{|l|c|c|c|c|c|c|c|}
\hline & I & II & III & IV & V & VI & VII \\
\hline Kryetari i bashkisë & Shykri & Sylejman & Shehsyvar & Shehsyvar & Shehsyvar & Shehsyvar & Shehsyvar \\
\hline Anëtar i bashkisë & Ahmet & $\begin{array}{c}\text { Abdurrahim } \\
\text { Zaimi }\end{array}$ & Musa & Musa & Musa & Musa & Musa \\
\hline Anëtar i bashkisë & Abdurrahman & Ibrahim Cërraga & Ymer & Ymer & Ymer & Hasan & Hasan \\
\hline Anëtar i bashkisë & Hysen & Musa Mezini & Ali & Ali & Ali & Kanber & Kanber \\
\hline Anëtar ibashkisë & $\begin{array}{c}\text { Djali i Numan } \\
\text { bejit }\end{array}$ & Hasan Domi & Ismail & Ismail & Ismail & Ismail & Ismail \\
\hline Anëtari bashkisë & $\begin{array}{c}\text { Losh papa } \\
\text { Mihal }\end{array}$ & $\begin{array}{c}\text { Losh papa } \\
\text { Mihal }\end{array}$ & SpiroXhufka & Spiro Xhufka & $\begin{array}{c}\text { Spiro } \\
\text { Xhufka }\end{array}$ & $\begin{array}{c}\text { Spiro } \\
\text { Xhufka }\end{array}$ & $\begin{array}{c}\text { Spiro } \\
\text { Xhufka }\end{array}$ \\
\hline Anëtar i bashkisë & Nosi Mim Nosi & Nosi Mim Nosi & Nos Mim Nosi & Ligor Popa & Ligor Popa & Ligor Papa & Ligor Papa \\
\hline $\begin{array}{l}\text { Sekretar dhe } \\
\text { arkëtar i bashkisë }\end{array}$ & XXX & XXX & Arif & Arif & Arif & Arif & Arif \\
\hline Mjeku i bashkisë & XXX & XXX & $\begin{array}{c}\text { Ismet } \\
\text { Myhedin }\end{array}$ & $\begin{array}{c}\text { Ismet } \\
\text { Myhedin }\end{array}$ & Ismet & Avram & Avram \\
\hline
\end{tabular}

Komisoni i arsimit

\begin{tabular}{|l|c|c|c|c|c|c|c|}
\hline & I & II & III & IV & V & VI & VII \\
\hline $\begin{array}{l}\text { Kryetari i } \\
\text { komisionit }\end{array}$ & XXX & XXX & Hakim & Hakim & Hakim & Hakim & Hakim \\
\hline Anëtar & XXX & XXX & Aqif & Aqif & Aqif & Aqif & Aqif \\
\hline Anëtar & XXX & XXX & Hysen & Hysen Hysni & Hysen Hysni & Hysen Hysni & Hysen Hysni \\
\hline
\end{tabular}




\begin{tabular}{|l|c|c|c|c|c|c|c|}
\hline & I & II & III & IV & V & VI & VII \\
\hline Anëtar & XXX & XXX & Haxhi Ali & Haxhi Ali & Haxhi Ali & Haxhi Ali & Haxhi Ali \\
\hline Anëtar & XXX & XXX & Mahmud Hamdi & Mahmud Hamdi & Mahmud & Mahmud & Mahmud \\
\hline Sekretar & XXX & XXX & Emin & Emin & Emin & Emin & Emin \\
\hline
\end{tabular}

Komisoni i vakëfeve

\begin{tabular}{|l|c|c|c|c|c|c|c|}
\hline & I & II & III & IV & V & VI & VII \\
\hline $\begin{array}{l}\text { Kryetari i komisionit } \\
\text { të vakëfeve }\end{array}$ & XXX & XXX & Hysen Hysni & Hysen Hysni & Hysen Hysni & Hysen Hysni & Hysen Hysni \\
\hline Anëtar & XXX & XXX & Haxhi Ali & Haxhi Ali & Haxhi Ali & Haxhi Ali & Haxhi Ali \\
\hline Anëtar & XXX & XXX & Sheh Myrteza & Hafiz Hysen & Hafiz Hysen & Hafiz Hysen & Hafiz Hysen \\
\hline Anëtar & XXX & XXX & Ibrahim & Ibrahim & Ibrahim & Mihal & Mihal \\
\hline Anëtar & XXX & XXX & Mihal & Musa & Musa & Musa & Musa \\
\hline Sekretar & XXX & XXX & Said & Said & Said & Said & Said \\
\hline
\end{tabular}

Nëpunësit e xhandarmërisë

\begin{tabular}{|l|c|c|c|c|c|c|c|}
\hline & I & II & III & IV & V & VI & VII \\
\hline Komandanti i taborit & XXX & Alush & Mihal & Haxhi Jashar & Jashar & Osman & Osman \\
\hline $\begin{array}{l}\text { Llogaritari i } \\
\text { xhandarmërisë }\end{array}$ & XXX & Mehmed Sami & Mehmet Sami & Mehmet Sami & Ali & Ali Riza & Demir \\
\hline Aga bylyku të parë & $\mathrm{XXX}$ & Xhafer & Xhafer & Mihal & Mihal & Mihal & Mihal \\
\hline $\begin{array}{l}\text { Ndihmës aga i } \\
\text { bylukut të parë }\end{array}$ & $\mathrm{XXX}$ & $\mathrm{XXX}$ & Iljas & Iljas & Iljas & Mihal & Osman \\
\hline Nëpunësi zhurnalit & $\mathrm{XXX}$ & $\mathrm{XXX}$ & Mehmed & Mehmed & Mehmed & Mehmed & Mehmed \\
\hline Aga i bylykut të dytë & $\mathrm{XXX}$ & Abdullah & Abdullah & Abdullah & Abdullah & Abdullah & Mihal \\
\hline $\begin{array}{l}\text { Ndihmës aga i bylykut } \\
\text { të dytë }\end{array}$ & $\mathrm{XXX}$ & Mihal & Mihal & Ibrahim & Ibrahim & Ibrahim & Ibrahim \\
\hline Emin zhurnali & $\mathrm{XXX}$ & Behram & Behram & Sylejman & Behram & Malik & Malik \\
\hline $\begin{array}{l}\text { Emini zhurnali } \\
\text { kalorësisë }\end{array}$ & $\mathrm{XXX}$ & Rexhep & Rexhep & Ismail & Ismail & Ismail & Ismail \\
\hline
\end{tabular}

Dega e bankës agrare të Elbasanit

\begin{tabular}{|l|c|c|c|c|c|c|c|}
\hline & I & II & III & IV & V & VI & VII \\
\hline $\begin{array}{l}\text { Kryetari i degës } \\
\text { bankare }\end{array}$ & XXX & XXX & Ibrahim & Ibrahim & Musa & Musa & Musa \\
\hline Nëpunësi i bankës & XXX & XXX & Mehmed Fikri & Mehmed Fikri & Fikri & Ibrahim & Mehmet Syrri \\
\hline Anëtar i bankës & XXX & XXX & Tahir & Tahir & Tahir & Tahir & Tahir \\
\hline
\end{tabular}




\section{Albanon}

Revistë kulturore

\begin{tabular}{|l|c|c|c|c|c|c|c|}
\hline & I & II & III & IV & V & VI & VII \\
\hline Ndihmës & XXX & XXX & Musa & Musa & Ryzhdi & Ryzhdi & Ryzhdi \\
\hline Sekretari & XXX & XXX & & Ali & Mihal & Salih & Salih \\
\hline Anëtar & XXX & XXX & Ali & XXX & Ymer & Ymer & Ymer \\
\hline Anëtar & XXX & XXX & Lush papa Mihal & & & & \\
\hline Anëtar & $X X X$ & $X X X$ & Ymer Adil & Jovan & $\begin{array}{c}\text { Spiro } \\
\text { Xhufka }\end{array}$ & $\begin{array}{c}\text { Spiro } \\
\text { Xhufka }\end{array}$ & Spiro Xhufka \\
\hline Taksidari bankës & $X X X$ & $X X X$ & Salih & Salih & Salih & Xhemali & Xhafer \\
\hline Odaxhi & $X X X$ & $X X X$ & $X X X$ & $X X X$ & $X X X$ & Hasan & Hasan \\
\hline
\end{tabular}

Drejtoria e detpublikut të Elbasanit

\begin{tabular}{|l|c|c|c|c|c|c|c|}
\hline & I & II & III & IV & V & VI & VII \\
\hline Nëpunësi & XXX & XXX & Davud Nijazi & Osman & Beqir & Jusuf & Jusuf \\
\hline Nëpunësi i hambarit & XXX & XXX & XXX & XXX & Ragip & Ragip & Ragip \\
\hline Shoqëruesi & XXX & XXX & XXX & Anonim & Abdyl & Hasan & Hasan \\
\hline
\end{tabular}

Drejtoria e depove të duhanit

\begin{tabular}{|l|c|c|c|c|c|c|c|}
\hline & I & II & III & IV & V & VI & VII \\
\hline Nëpunësi & XXX & XXX & Xhafer & Xhafer & Xhafer & Hasan & Hasan \\
\hline Shoqëruesi & XXX & XXX & XXX & Anonim & Haxhi & Haxhi & Haxhi \\
\hline Shoqëruesi & XXX & XXX & XXX & Anonim & Adem & Adem & Adem \\
\hline
\end{tabular}

Shkolla ruzhdije (tetëvjeçare)

\begin{tabular}{|l|c|c|c|c|c|c|c|}
\hline & I & II & III & IV & V & VI & VII \\
\hline Mësuesi i parë & Hysen & Hysen & Hysen Hysni & Hysen Hysni & Hysen Hysni & Hysen Hysni & Hysen Hysni \\
\hline Mësuesi i dytë & $\begin{array}{c}\text { Hafiz } \\
\text { Sylejman }\end{array}$ & $\begin{array}{c}\text { Hafiz } \\
\text { Sylejman }\end{array}$ & $\begin{array}{c}\text { Hafiz } \\
\text { Sylejman }\end{array}$ & $\begin{array}{c}\text { Hafiz } \\
\text { Sylejman }\end{array}$ & $\begin{array}{c}\text { Hafiz } \\
\text { Sylejman }\end{array}$ & $\begin{array}{c}\text { Hafiz } \\
\text { Sylejman }\end{array}$ & $\begin{array}{c}\text { Hafiz } \\
\text { Sylejman }\end{array}$ \\
\hline Mësuesi i rikasë & XXX & XXX & Said & Said & Said & Said & Said \\
\hline Portieri & Ibrahim & XXX & XXX & Ibrahim & Ibrahim & Ibrahim & Ibrahim \\
\hline
\end{tabular}

Mësuesi i shkollës iptidaije (fillore)

\begin{tabular}{|l|c|c|c|c|c|c|c|}
\hline & I & II & III & IV & V & VI & VII \\
\hline Mësuesi i parë & XXX & Adem & Adem & Adem & Adem & Adem & Adem \\
\hline Mësuesi i dytë & XXX & Ali & Ali & Ali & Ali & Ali & Ali \\
\hline Portieri & XXX & XXX & XXX & Selim & Memish & Memish & Memish \\
\hline
\end{tabular}


Të dhënat demografike

\begin{tabular}{|c|c|c|c|c|c|c|c|}
\hline Kaza & Fshati & Banesa & Banorë & Nahija & Fshati & Banesa & Banorë \\
\hline & Elbasan & 1696 & 5015 & & Shpat & 10 & 20 \\
\hline & $\begin{array}{l}\text { Allaj } \\
\text { begalide }\end{array}$ & 7 & 20 & & Stërstanj & 9 & 16 \\
\hline & Ollma & 13 & 59 & & Shtëpanj & 12 & 16 \\
\hline & Shtëmaj & 16 & 35 & & Shtërmen & 46 & 109 \\
\hline & İraguzhda & 20 & 20 & & Beshdan & 4 & 10 \\
\hline & Skroska & 16 & 16 & & Polis & 96 & 331 \\
\hline & Bulqiza & 10 & 26 & & Pashtaresh & 10 & 24 \\
\hline & Broshka & 22 & 42 & & Çiburesh & 5 & 8 \\
\hline & Belsh & 142 & 535 & & Çigar & 4 & 5 \\
\hline & Biçakanj & 2 & 4 & & Çikalesh & 2 & 6 \\
\hline & Baltza & 44 & 128 & & Çigzoja & 2 & 11 \\
\hline & Plangarica & 11 & 53 & & Hajdaranj & 3 & 4 \\
\hline & Bzhuta & 6 & 17 & & Driza & 1 & 7 \\
\hline & Bradashesh & 41 & 79 & & Zavalina & 15 & 40 \\
\hline & Bodin & 25 & 65 & & Selita & 14 & 35 \\
\hline & Biza & 17 & 38 & & Shushica & 34 & 80 \\
\hline & Bishqem & 73 & 259 & & Shelcan & 18 & 21 \\
\hline & Bixulla & 24 & 118 & & Shlbatra & 8 & 15 \\
\hline & Branesh & 3 & 13 & & Shënavlash & 10 & 17 \\
\hline & Buzgan & 5 & 7 & & $\begin{array}{l}\text { Shushica } \\
\text { kadi }\end{array}$ & 5 & 14 \\
\hline & Paksht & 27 & 66 & & Shergjan & 12 & 19 \\
\hline & Pajanga & 26 & 95 & & Todan & 4 & 6 \\
\hline & Papr Solak & 2 & 7 & & Galikat & 6 & 16 \\
\hline & Patresh & 27 & 57 & & Gostima & 10 & 28 \\
\hline & Pajova & 32 & 82 & & Kaçol & 6 & 14 \\
\hline & Pajon & 23 & 116 & & Kodra Bujas & 6 & 8 \\
\hline & Prapaniga & 31 & 78 & & Kuqan & 11 & 56 \\
\hline & $\begin{array}{l}\text { Preça } \\
\text { poshtme }\end{array}$ & 9 & 30 & & Kryezjarth & 6 & 9 \\
\hline & $\begin{array}{l}\text { Preça e } \\
\text { sipërme }\end{array}$ & 5 & 14 & & Gjinar & 16 & 47 \\
\hline
\end{tabular}


Revistë kulturore

\begin{tabular}{|c|c|c|c|c|c|c|c|}
\hline Kaza & Fshati & Banesa & Banorë & Nahija & Fshati & Banesa & Banorë \\
\hline & Pishkash & 19 & 19 & & Gjorgjovina & 2 & 8 \\
\hline & Trepsenisht & 5 & 6 & & Gjergjan & 30 & 100 \\
\hline & Tregan & 8 & 13 & & Lukan & 5 & 10 \\
\hline & Çuburake & 21 & 44 & & Leshan & 11 & 25 \\
\hline & Cërrik & 39 & 101 & & Mliza & 6 & 6 \\
\hline & Çiraga & 7 & 18 & & Muçanj & 2 & 4 \\
\hline & Çardalos & 3 & 22 & & Mallasenj & 10 & 20 \\
\hline & Cacabez & 42 & 195 & & Mjekras & 6 & 6 \\
\hline & Çuburake & 35 & 89 & & Nezhan & 4 & 4 \\
\hline & Çërruja & 46 & 232 & & Valsh & 15 & 48 \\
\hline & Haxhi Alija & 3 & 7 & & Joranisht & 6 & 13 \\
\hline & Hasmakanj & 2 & 5 & & Çermenika & 37 & 92 \\
\hline & Hotosht & 10 & 10 & & Orhenk & 37 & 92 \\
\hline & Dragot & 23 & 76 & & Floq & 24 & 38 \\
\hline & Dishiranj & 9 & 26 & & İzdargjesha & 33 & 72 \\
\hline & Dushkanj & 7 & 20 & & Panezh & 4 & 5 \\
\hline & Dors & 33 & 35 & & Shmil & 188 & 210 \\
\hline & Dragostunja & 25 & 25 & & Gurakuq & 88 & 155 \\
\hline & Raha (Rasa) & 54 & 168 & & Kotraman & 20 & 38 \\
\hline & Rens & 10 & 37 & & Kostinja & 36 & 60 \\
\hline & Reçanj & 8 & 18 & & Lumnik & 30 & 64 \\
\hline & Rrilla & 10 & 29 & & Martanesh & 125 & 125 \\
\hline & Zerja & 12 & 22 & & Neshta & 22 & 41 \\
\hline & Seferenj & 18 & 82 & & & & \\
\hline & Seriça & 41 & 94 & & & & \\
\hline & Shez Kelshika & 20 & 38 & & & & \\
\hline & Shalës & 41 & 128 & & & & \\
\hline & Shingjin & 13 & 42 & & & & \\
\hline & Shalqinas & 13 & 24 & & & & \\
\hline & Shmhil & 7 & 29 & & & & \\
\hline & Shëngjon & 14 & 40 & & & & \\
\hline & Sadi beganj & 6 & 45 & & & & \\
\hline & Tatrash & 21 & 41 & & & & \\
\hline
\end{tabular}




\begin{tabular}{|c|c|c|c|c|c|c|c|}
\hline Kaza & Fshati & Banesa & Banorë & Nahija & Fshati & Banesa & Banorë \\
\hline & Tërbaç & 28 & 85 & & & & \\
\hline & Todoranj & 10 & 38 & & & & \\
\hline & Aligjinaj & 8 & 25 & & & & \\
\hline & Gryksh & 16 & 56 & & & & \\
\hline & Guras & 7 & 17 & & & & \\
\hline & Grekan & 9 & 40 & & & & \\
\hline & Gracen & 9 & 30 & & & & \\
\hline & Garunja & 32 & 100 & & & & \\
\hline & Godolesh & 92 & 250 & & & & \\
\hline & Griqan & 18 & 40 & & & & \\
\hline & Gurabarth & 5 & 23 & & & & \\
\hline & Gurazez & 29 & 156 & & & & \\
\hline & Gizavesh & 29 & 29 & & & & \\
\hline & Fag & 30 & 226 & & & & \\
\hline & Kajan & 10 & 32 & & & & \\
\hline & Kozanj & 11 & 30 & & & & \\
\hline & Kus & 6 & 24 & & & & \\
\hline & Kara kullak & 6 & 20 & & & & \\
\hline & Kulla Papri & 6 & 16 & & & & \\
\hline & Kokonja & 32 & 72 & & & & \\
\hline & Koxhanj & 8 & 30 & & & & \\
\hline & Kusarth & 13 & 29 & & & & \\
\hline & Kollojka & 12 & 146 & & & & \\
\hline & Kore & 14 & 115 & & & & \\
\hline & Kokrava & 6 & 6 & & & & \\
\hline & Gjyrala & 15 & 52 & & & & \\
\hline & Gjonm & 12 & 46 & & & & \\
\hline & Luga & 11 & 34 & & & & \\
\hline & Lazaranj & 11 & 27 & & & & \\
\hline & Lashpot & 128 & 398 & & & & \\
\hline & Letan & 11 & 41 & & & & \\
\hline & Liporash & 33 & 33 & & & & \\
\hline & Moriqan & 31 & 134 & & & & \\
\hline
\end{tabular}




\section{Albanon \\ Revistë kulturore}

\begin{tabular}{|c|c|c|c|c|c|c|c|}
\hline Kaza & Fshati & Banesa & Banorë & Nahija & Fshati & Banesa & Banorë \\
\hline & Malkas & 5 & 10 & & & & \\
\hline & Murris & 16 & 32 & & & & \\
\hline & $\begin{array}{l}\text { Mustafa } \\
\text { Koçanj }\end{array}$ & 16 & 46 & & & & \\
\hline & Miraka & 41 & 97 & & & & \\
\hline & Molagjesh & 23 & 96 & & & & \\
\hline & Vidhas & 13 & 24 & & & & \\
\hline & $\begin{array}{l}\text { Vidhas } \\
\text { Hasgjel }\end{array}$ & 28 & 35 & & & & \\
\hline & Veskanji & 24 & 68 & & & & \\
\hline & Velçan & 6 & 6 & & & & \\
\hline & Jatesh & 12 & 46 & & & & \\
\hline
\end{tabular}

